\title{
Avaliação Seriada versus Exame Vestibular: Semelhanças e Diferenças entre Coortes no Curso de Medicina da Universidade de Brasília
}

\author{
Serial Evaluation versus Admission Exam: \\ Similarities and Differences between \\ Cohorts in the Medical Program at the \\ University of Brasilia
}

PALAVRAS-CHAVE:

- Educação Médica;

- Critério de Admissão;

- Avaliação Educacional;

- Estudantes de Medicina;

- Aprendizagem.

KEY-WORDS

- Medical Education;

- School Admission Criteria;

- Educational Measurement;

- Medical Students;

- Learning.

Recebido em: 29/11/2005

Reencaminhado em: 04/05/2006

Aprovado em: 07/11/2006

\section{RESUMO}

O propósito deste estudo retrospectivo foi avaliar o impacto do processo de avaliação seriada, em comparação com exame vestibular, em termos de efeitos no perfil de atributos e de progresso acadêmico de alunos da Universidade de Brasília. Os sujeitos foram 415 alunos (57,8\% masculinos) registrados no Curso de Medicina após aprovação para ingresso mediante avaliação seriada, ou exame vestibular no primeiro ou segundo semestre, ao longo de seis anos (1999-2004). Foram comparados, entre os grupos de ingresso, os indicadores demográficos, de atitudes em face do aprendizado e de rendimento cognitivo no curso. Os resultados mostraram proporções significantemente maiores de alunos naturais do Distrito Federal e da faixa etária mais jovem no grupo da avaliação seriada. O índice de rendimento acadêmico manteve-se significativamente mais elevado nesse grupo desde o segundo ao nono período do curso. Análises estatísticas indicam que tal efeito reflete, além da forma e do ano de ingresso, outras diferenças nas características dos alunos, tais como sexo, idade e valoração do aprendizado. Os achados sugerem que a avaliação seriada privilegiou a aptidão acadêmica quanto ao rendimento cognitivo e alterou tendências na composição demográfica do alunado com efeitos no próprio rendimento estudantil.

\begin{abstract}
The purpose of this retrospective study was to appraise the impact of a new admission process (serial evaluation) on the attributes and achievement patterns of medical students in comparison to the standing onetime admission test. The subjects were 415 students (57,8\% males) enrolled in the medical program of the University of Brasilia, admitted through serial evaluation or admission test in the course of six years (1999-2004). The admission groups were compared as refers to demographic data, learning attitudes and academic achievement indicators. The results showed significantly higher proportions of students born in the Federal District and of a younger age-bracket in the serial evaluation group than in comparison to the admission test group. Academic achievement was also significantly higher in this group, from the second to the ninth semester. Statistical analyses indicate that besides the year and the criteria of admission the effect on academic achievement reflected other differences such as sex, age and self-appraisal of learning outcomes in the features of the students. These findings suggest that the new admission process has favored academic aptitude in terms of cognitive achievement. It also seems to have modified the demographic composition of the student body with effects on academic achievement in itself.
\end{abstract}




\section{INTRODUÇÃO}

O processo de seleção de candidatos ao Curso de Medicina tem implicações que extrapolam o simples desfecho de acesso ao campo profissional. Numa ampla revisão das diretrizes e práticas desse processo em âmbito internacional, McGaghie avalia que a seleção de candidatos tem propósitos de curto e de longo prazos. No primeiro caso, as metas dizem respeito ao progresso dos alunos no próprio curso; no segundo, se referem à contribuição dos egressos para provisão de assistência médica, melhoria da saúde pública, apoio ao ensino e à pesquisa e exercício de outras atividades de promoção da profissão ${ }^{1}$.

No Brasil, o processo de seleção para acesso ao ensino superior tem caráter institucional derivado de diretrizes federais, as quais visam ao reconhecimento do mérito acadêmico sem viés subjetivo ${ }^{2}$. O processo focaliza o critério de preparo educacional, apurado em concurso de provas escritas que abrangem múltiplas matérias no denominado exame vestibular. A classificação de rendimento obtido nesse exame determina o preenchimento de cota fixa de candidatos, isto é, do número de vagas oferecido pela instituição.

O exame vestibular é alvo de críticas, especialmente por ocorrer em um único momento e por apresentar influência negativa sobre o ciclo que o antecede, o segundo grau. Por conta desse último senão, além de não gerar informações que contribuam para aperfeiçoar o desempenho individual do aluno e o processo educacional das escolas de ensino médio e não contribuir para a definição de políticas para esse nível de ensino, transforma, para muitos alunos e escolas, esse período de tempo numa preparação precípua para determinada prova $^{3}$.

Para enfrentar essas questões, já em meados da década de 1980, algumas Instituições de Ensino Superior (IES), entre as quais sobressaem a Fundação Cesgranrio e a Universidade de Brasília (UnB), iniciaram discussões para implantar mecanismos que avaliassem os alunos ao longo do curso secundário. A Cesgranrio conseguiu autorização ministerial para implementar, entre 1992 e 1995, o Sistema de Avaliação Progressiva para Ingresso no Ensino Superior (Sapiens). Em 1995, a UnB reinicia seus estudos sobre alternativas ao vestibular e implanta, em 1996, o Programa de Avaliação Seriada (PAS). Também em 1995, a Universidade Federal de Santa Maria desenvolve o Programa de Ingresso ao Ensino Superior (Peies), e outras IES, como a Universidade Federal de Uberlândia, Universidade Federal de Viçosa e Universidade do Amazonas, dão início a estudos e programas semelhantes ${ }^{3}$.

O arcabouço legal que dá sustentação à adoção de processos alternativos de seleção para o terceiro grau encontra-se na Lei 9.394/96, que estabelece as Diretrizes e Bases da Educação Nacional, e no Parecer no CP 98/99, aprovado em 06/07/ 99, que trata da Regulamentação de Processo Seletivo para acesso a cursos de graduação de Universidades, Centros Universitários e Instituições Isoladas de Ensino Superior que facultam às IES a adoção de modelos individualizados ${ }^{2,4}$.

$\mathrm{Na}$ UnB, a seleção e o ingresso de novos alunos ocorrem semestralmente há mais de três décadas mediante dois vestibulares anuais. O processo de avaliação seriada foi implantado em 1996, após discussão e aprovação pelos colegiados da instituição. Desde 1999, quando foi admitida a primeira turma, metade das vagas ofertadas para novos alunos no primeiro semestre de cada ano é reservada a essa modalidade de seleção. No PAS, o aluno matriculado no segundo grau é avaliado, ao final do ano, por três anos consecutivos. Entre outros objetivos específicos, o processo visa a uma avaliação da aprendizagem significativa, que privilegie a reflexão ao invés da memorização, a qualidade sobre a quantidade de informações, o ensino sobre o adestramento, o processo sobre o produto $^{5}$.

Alterações em critérios de seleção podem gerar impactos diversos que são passíveis de acompanhamento por processo de auditagem interna ${ }^{1,6}$. A instigação para nosso estudo foi a necessidade percebida de avaliar o impacto do processo alternativo de seleção, à luz dos objetivos do PAS, ao se formar a primeira turma de médicos que incluiu o ingresso por avaliação seriada. Alunos que ingressam mediante avaliação seriada diferem em atributos e progresso acadêmico daqueles que ingressam por exame vestibular? Essa questão norteou o presente trabalho, que examina os seguintes objetivos de comparação entre os distintos grupos de ingresso:

- Determinar a extensão das eventuais diferenças nas características demográficas dos alunos, inclusive por cotejo com época anterior ao estudo;

- Verificar o nível das diferenças quanto ao rendimento no aprendizado e às atitudes dos alunos sobre a aprendizagem ao término do primeiro ano de curso;

- Averiguar, no acompanhamento subseqüente, o sentido das diferenças quanto ao progresso dos alunos no curso em termos de rendimento e evasão.

\section{MÉTODOS}

\section{Delineamento}

Trata-se de estudo retrospectivo, de tipo causal-comparativo, constituído de dados de 12 coortes (turmas de ingresso semestral) consecutivas com elementos de acompanhamento por dois, seis ou nove semestres. 


\section{Sujeitos}

Os sujeitos do estudo foram 415 alunos (57,8\% do sexo masculino) registrados no Curso de Medicina da Universidade de Brasília ao longo de seis anos (1999-2004), após aprovação para ingresso mediante avaliação seriada - 99 (23,9\%) ou exames vestibulares semestrais. A média de idade deste alunado era 19 anos e dois meses ( $\mathrm{dp}=19$ meses), ao término do semestre de ingresso. Alunos transferidos de outras instituições não integraram o conjunto de sujeitos.

A amostra da análise de rendimento incluiu 400 alunos (56,8\% do sexo masculino). Houve perda de 15 alunos - dos quais 12 ingressaram por vestibular - em razão de evasão, seja por desistência imediata, ou por abandono ou exclusão antes da integralização dos créditos do primeiro ano do curso.

\section{Contexto}

O ingresso no curso é semestral. Na época do estudo, metade das vagas ofertadas no primeiro semestre de cada ano foi reservada para seleção por avaliação seriada, e o restante para seleção pelo primeiro exame vestibular; as vagas ofertadas no segundo semestre continuaram a ser destinadas exclusivamente à seleção pelo segundo exame vestibular. Ambos os processos de seleção estão abertos a alunos residentes em qualquer município mediante inscrição individual, mas a inscrição para a avaliação seriada exige que o candidato esteja institucionalmente matriculado no segundo grau.

Todos os alunos seguiram o currículo implantado em 1988, que privilegia a formação geral e tem caráter semi-seriado, matrícula por disciplina semestral e regime de créditos. O fluxo curricular não foi alterado no período 1999-2004, mas o alunado incluso no estudo superou o alunado da época anterior de igual duração $(\mathrm{N}=415$ vs. $\mathrm{N}=357)$, por conta do aumento de vagas para ingresso semestral.

\section{Medidas e procedimentos}

As duas fontes de informação para o estudo foram: (1) o registro acadêmico do aluno, que inclui dados demográficos, tipo de admissão e histórico escolar; (2) o inventário de condições e expectativas, preenchido pelo aluno no início do terceiro período do curso e que abrange distintos descritores de atitudes do aprendiz em face do aprendizado no primeiro ano. Os diversos indicadores envolvidos foram medidos como descrito a seguir.

Rendimento cognitivo. Mediu-se esse indicador por meio do índice de rendimento acadêmico (IRA). Na Universidade de Brasília, esse índice serve de critério de ordenação para obtenção de vagas na matrícula semestral em disciplinas e sintetiza a progressão acadêmica do aluno. O IRA tem caráter cumulativo e seu cálculo reflete as menções ponderadas pelos respectivos números de créditos das disciplinas cursadas, bem como a permanência do aluno no fluxo do curso. A faixa de pontuação varia de zero a cinco. Neste estudo, apurou-se o IRA cumulativo registrado ao término do segundo, do sexto e do nono períodos do fluxo curricular do curso.

Questionário de Valoração do Curso (QVC). O QVC é um instrumento de 36 itens, adaptado do Course Valuing Inventory ${ }^{7}$ de Nehari e Bender, mediante versão em português e modificação da terminologia para refletir a perspectiva da vivência curricular global e não somente de uma disciplina isolada ${ }^{8}$. O instrumento serviu como indicador das percepções dos alunos sobre o valor e o significado dos desfechos de aprendizado no primeiro ano do curso. A consistência interna das respostas na amostra de análise foi altamente satisfatória (alfa de Cronbach $=0,94$ ). A faixa de pontuação varia de 36 a 144. Quatro itens representativos do QVC (e respectivas correlações item-total corrigidas) são os seguintes:

- Item 20: o curso até aqui foi uma vivência de aprendizagem construtiva e definitivamente proveitosa $(r=0,665)$;

- Item 24: lidei, de algum modo, com mais desafios nas disciplinas do curso e estou satisfeito por isso ( $r=0,647)$;

- Item 29: não me foi possível interligar as noções e fazer muito sentido do conteúdo apresentado nas disciplinas anteriores ( $r=0,636$ - polaridade invertida);

- Item 35: as disciplinas do curso não me ajudaram a adquirir um conhecimento cuidadoso das matérias $(\mathrm{r}=$ 0,632 - polaridade invertida).

Escalas analógicas. Autoconfiança como aprendiz e motivação global para prosseguir os estudos foram aferidos por escalas analógicas visuais de $100 \mathrm{~mm}$. A faixa de pontuação varia de 0 a 100 para ambos os indicadores.

Procedimentos. O QVC e as escalas analógicas foram aplicados na primeira semana do terceiro período letivo do curso, como parte do inventário de condições e expectativas. Esse material tinha a natureza da experiência inicial no curso como objeto de referência e visava propiciar reflexão sobre a vivência educacional e as expectativas quanto ao prosseguimento do curso. Os dados foram obtidos com asseguramento de confidencialidade pessoal, e as respostas coletivas foram comentadas em cada turma no contexto de orientação educativa. Cópias dos instrumentos utilizados podem ser fornecidas mediante solicitação ao endereço de correspondência.

\section{Análise}

Os textos de Fraenkel e Wallen e de Howell orientaram os planos e procedimentos de análise $\mathrm{e}^{9,10}$. Três procedimen- 
tos estatísticos principais foram utilizados: (a) testes $\mathrm{t}$ ou análises de variância, para medir diferenças entre médias de grupos; (b) testes de qui-quadrado, para comparar características ou descritores entre grupos; (c) testes do Modelo Linear Geral (MLG), para aferir efeitos de distintos grupamentos dos participantes no índice de rendimento acadêmico ajustado por co-variáveis. O nível de significância foi fixado em $\mathrm{P}<0,05$. A análise foi realizada no programa SPSS 12.0.

\section{RESULTADOS}

A Tabela 1 mostra a distribuição dos alunos da época do estudo segundo a forma e o ano de ingresso na universidade, entre 1999 e 2004. Não houve diferenças significantes na distribuição de sexo, faixa etária e naturalidade no decurso de seis anos (teste de qui-quadrado, menor $\mathrm{P}=0,673$ ). A evasão (15 alunos) ainda no primeiro ano do curso apresentou taxas similares entre os grupos de exame vestibular (3,8\%) e de avaliação seriada (3,0\%). Acrescente-se que a evasão subseqüente foi menor (5 alunos, ou 2,5\%) no acompanhamento das seis primeiras turmas $(\mathrm{N}=198)$, do terceiro ao nono períodos letivos.

TABELA 1

Distribuição de alunos de Medicina por forma de ingresso e evasão no primeiro ano de estudos conforme o ano de ingresso na Universidade de Brasília $(\mathrm{N}=415)$

\begin{tabular}{|c|c|c|c|c|}
\hline Ano de ingresso & $\begin{array}{l}\text { Forma de Ingresso } \\
\text { Avaliação Seriada }\end{array}$ & $1^{0}$ vestibular & $2^{\circ}$ vestibular & $\begin{array}{l}\text { Evasão* }^{*} \\
1^{\mathrm{o}} \text { ano }\end{array}$ \\
\hline $1(\mathrm{n}=57)$ & 12 & 15 & 30 & 0 \\
\hline $2(\mathrm{n}=71)$ & 16 & 20 & 35 & 1 \\
\hline $3(\mathrm{n}=73)$ & 18 & 19 & 36 & 2 \\
\hline $4(\mathrm{n}=70)$ & 18 & 18 & 34 & 2 \\
\hline $5(\mathrm{n}=72)$ & 18 & 18 & 36 & 5 \\
\hline $6(\mathrm{n}=72)$ & 17 & 19 & 36 & 5 \\
\hline Total $(\mathrm{N}=415)$ & 99 & 109 & 207 & 15 \\
\hline
\end{tabular}

* Doze casos de ingresso por vestibulares e três por avaliação seriada.

Comparações das características do alunado do estudo com alunados de épocas anteriores, a partir da implantação do atual currículo em 1988, revelaram proporções significativamente crescentes de indivíduos naturais do Distrito Federal e de faixa etária maior que 18 anos. Não houve tendência significativa no que tange à distribuição sexual, embora se note aumento da parcela feminina no alunado do estudo em comparação com o alunado da época anterior de igual duração (Tabela 2). 
TABELA 2

Distribuição de alunos de Medicina conforme atributos demográficos e época de ingresso na Universidade de Brasília $(\mathrm{N}=994)$

\begin{tabular}{|c|c|c|c|c|c|}
\hline \multirow{2}{*}{$\begin{array}{c}\text { Atributos } \\
\text { demográficos }\end{array}$} & \multirow{2}{*}{$\begin{array}{c}\text { Época de ingresso } \\
\begin{array}{c}1988-1992 \\
n=222(\%)\end{array}\end{array}$} & \multirow{2}{*}{$\begin{array}{c}1993-1998 \\
n=357(\%)\end{array}$} & \multirow{2}{*}{$\begin{array}{c}1999-2004 \\
n=415(\%)\end{array}$} & \multicolumn{2}{|c|}{ Teste de Associação } \\
\hline & & & & Phi & $\mathbf{P}$ \\
\hline $\begin{array}{l}\text { Sexo }^{\text {a }} \\
\text { Masculino } \\
\text { Feminino }\end{array}$ & $\begin{array}{r}124(55,9) \\
98(44,1)\end{array}$ & $\begin{array}{l}222(62,2) \\
135(37,8)\end{array}$ & $\begin{array}{l}240(57,8) \\
175(42,2)\end{array}$ & 0,051 & 0,268 \\
\hline $\begin{array}{l}\text { Faixa etária }^{\mathrm{b}} \\
\text { Menos de } 19 \text { anos } \\
\text { Mais de } 18 \text { anos }\end{array}$ & $\begin{array}{r}131(59,0) \\
91(41,0)\end{array}$ & $\begin{array}{l}168(47,1) \\
189(52,9)\end{array}$ & $\begin{array}{l}162(39,0) \\
253(61,0)\end{array}$ & 0,151 & 0,000 \\
\hline $\begin{array}{l}\text { Naturalidadec }^{c} \\
\text { Distrito Federal } \\
\text { Outro local }\end{array}$ & $\begin{array}{r}88(39,6) \\
134(60,4)\end{array}$ & $\begin{array}{l}207(58,0) \\
150(42,0)\end{array}$ & $\begin{array}{l}282(68,0) \\
133(32,0)\end{array}$ & 0,214 & 0,000 \\
\hline
\end{tabular}

Testes qui-quadrado para tendência (grau de liberdade $=1$ ):

(a) Qui-quadrado $=0,03 \mathrm{P}=0,854$.

(b) Qui-quadrado $=22,9 \mathrm{P}<0,0001$.

(c) Qui-quadrado $=46,0 \mathrm{P}<0,0001$.

A Tabela 3 revela as características demográficas dos alunos da época do estudo, segundo a forma de ingresso. Foram observadas diferenças significativas entre os grupos de ingresso na distribuição dos participantes quanto à faixa etária e naturalidade, mas não quanto ao sexo. São significantemente maiores no grupo de avaliação seriada, em comparação com os grupos do primeiro e do segundo exame vestibular, as proporções tanto de alunos nascidos no Distrito Federal quanto daqueles pertencentes à faixa etária mais jovem no semestre de ingresso.

TABELA 3

Distribuição de alunos de Medicina conforme atributos demográficos e forma de ingresso na Universidade de Brasília, no período de 1/1999 a 2/2004 (N = 415)

\begin{tabular}{|c|c|c|c|c|c|}
\hline \multirow{2}{*}{$\begin{array}{c}\text { Atributos } \\
\text { demográficos }\end{array}$} & \multirow{2}{*}{$\begin{array}{c}\text { Forma de ingresso } \\
\text { Avaliação seriada } \\
n=99(\%)\end{array}$} & \multirow{2}{*}{$\begin{array}{c}1^{o} \text { Vestibular } \\
\mathrm{n}=108(\%)\end{array}$} & \multirow{2}{*}{$\begin{array}{c}2^{\circ} \text { Vestibular } \\
\mathrm{n}=208(\%)\end{array}$} & \multicolumn{2}{|c|}{ Teste de Associação } \\
\hline & & & & Phi & $\mathbf{P}$ \\
\hline $\begin{array}{l}\text { Sexo }^{\mathrm{a}} \\
\text { Masculino } \\
\text { Feminino }\end{array}$ & $\begin{array}{l}53(53,1) \\
46(46,9)\end{array}$ & $\begin{array}{l}60(53,9) \\
48(46,1)\end{array}$ & $\begin{array}{r}127(59,9) \\
81(40,1)\end{array}$ & 0,064 & 0,393 \\
\hline $\begin{array}{l}\text { Faixa etária }^{\mathrm{b}} \\
\text { Menos de } 19 \text { anos } \\
\text { Mais de } 18 \text { anos }\end{array}$ & $\begin{array}{r}97(97,9) \\
2(2,1)\end{array}$ & $\begin{array}{l}21(20,6) \\
87(79,4)\end{array}$ & $\begin{array}{r}44(19,8) \\
164(80,2)\end{array}$ & 0,683 & 0,000 \\
\hline $\begin{array}{l}\text { Naturalidade }{ }^{c} \\
\text { Distrito Federal } \\
\text { Outro local }\end{array}$ & $\begin{array}{l}84(86,5) \\
15(13,5)\end{array}$ & $\begin{array}{l}75(72,5) \\
33(27,5)\end{array}$ & $\begin{array}{r}123(58,9) \\
85(41,1)\end{array}$ & 0,244 & 0,000 \\
\hline
\end{tabular}


Não foram observadas diferenças significativas entre os três grupos de ingresso no tocante a duas medidas de atitudes em face do aprendizado no primeiro ano de estudos: escore total do Questionário de Valoração do Curso (QVC) e grau de motivação global para prosseguir nos estudos. Houve diferença pouco expressiva, mas significante entre os grupos no nível de autoconfiança como aprendiz. A Tabela 4 mostra os valores obtidos.

TABELA 4

Diferenças após o primeiro ano de estudos de médias no escore do Questionário de Valoração do Curso (QVC), no nível de autoconfiança como aprendiz e no grau de motivação global para prosseguir nos estudos, entre alunos de Medicina agrupados segundo a forma de ingresso no curso $(\mathrm{N}=400)^{*}$

\begin{tabular}{|c|c|c|c|}
\hline Forma de ingresso & $\begin{array}{c}\text { Medidas } \\
\text { Valoração do Curso } \\
\text { Média }(\mathrm{dp})^{\mathrm{a}}\end{array}$ & $\begin{array}{l}\text { Autoconfiança } \\
\text { 2. Média }(d p)^{b}\end{array}$ & $\begin{array}{l}\text { Motivação global } \\
\text { Média (dp) }\end{array}$ \\
\hline Avaliação seriada $(n=96)$ & $117,7(12,2)$ & $73,4(18,8)$ & $86,2(15,9)$ \\
\hline Primeiro vestibular $(n=102)$ & $119,1(12,4)$ & $76,8(15,2)$ & $90,1(12,0)$ \\
\hline Segundo vestibular $(n=202)$ & $118,1(14,9)$ & $78,8(15,5)$ & $87,7(17,5)$ \\
\hline
\end{tabular}

* Análise de variância (one-way) por forma de ingresso. Teste robusto de igualdade de médias (Welch, estatística F de distribuição assintótica).

(a) $\mathrm{F}=0,3 \mathrm{df}(2 ; 223,9)$ significância $=0,732$.

(b) $\mathrm{F}=3,1 \mathrm{df}(2 ; 200,5)$ significância $=0,048$.

(c) $\mathrm{F}=2,0 \mathrm{df}(2 ; 224,5)$ significância $=0,131$.

Já o IRA, como indicador de rendimento cognitivo alcançado ao término do primeiro ano, diferiu significativamente entre os grupos de ingresso (Anova, F $(2,397)=16,2 \mathrm{P}<$ 0,001). Um teste post-hoc de comparação múltipla (Tukey HSD) mostra que as diferenças entre as médias dos três grupos foram significantemente distintas entre si. A magnitude da diferença entre as médias foi menor na comparação de avaliação seriada com o primeiro vestibular (tamanho do efeito, $\mathrm{ES}=0,38$ ) do que na comparação entre avaliação seriada e o segundo vestibular (tamanho do efeito, ES =0,74). Análises por subgrupos de sexo, faixa etária e naturalidade revelaram discrepâncias importantes nas diferenças entre as três formas de ingresso. As diferenças mais expressivas no IRA ocorreram nos subgrupos de naturais do Distrito Federal e de ambos os sexos. Não houve, entretanto, diferenças significantes entre as formas de ingresso nos subgrupos de faixa etária menor de 19 anos e de outra naturalidade. Nos quatro casos em que a diferença entre médias foi significante, o grupo de avaliação seriada apresentou índice de rendimento mais elevado (Tabela 5). 
TABELA 5

Análise de regressão (Modelo Linear Geral). Efeitos de percepções do valor do aprendizado (QVC) no Índice de Rendimento Acadêmico (IRA) ao término do $1^{\circ}$ ano entre alunos de Medicina agrupados por sexo, faixa etária, naturalidade e tipo de ingresso no curso $(\mathrm{n}=400)$

\begin{tabular}{|c|c|c|c|c|c|c|}
\hline Subgrupos & $\mathbf{N}$ & $\begin{array}{c}\text { IRA } \\
\text { Avaliação seriada } \\
\text { Média (dp) }\end{array}$ & $\mathbf{N}$ & $\begin{array}{l}1^{o} \text { vestibular } \\
\text { Média (dp) }\end{array}$ & $\mathbf{N}$ & $\begin{array}{l}\text { 20 vestibular } \\
\text { Média (dp) }\end{array}$ \\
\hline $\begin{array}{l}\text { Sexo } \\
\text { Masculino }^{\mathrm{a}} \\
\text { Feminino }^{\mathrm{b}}\end{array}$ & $\begin{array}{l}51 \\
45\end{array}$ & $\begin{array}{l}3,97(0,43) \\
4,21(0,36)\end{array}$ & $\begin{array}{l}55 \\
47\end{array}$ & $\begin{array}{l}3,82(0,42) \\
4,06(0,33)\end{array}$ & $\begin{array}{r}121 \\
81\end{array}$ & $\begin{array}{l}3,74(0,37) \\
3,91(0,33)\end{array}$ \\
\hline $\begin{array}{l}\text { Faixa etária } \\
\text { < de } 19 \text { anos }^{\mathrm{c}} \\
>\text { de } 18 \text { anos }^{\mathrm{d}}\end{array}$ & $\begin{array}{r}94 \\
2\end{array}$ & $\begin{array}{l}4,08(0,42) \\
4,02(0,03)\end{array}$ & $\begin{array}{l}21 \\
81\end{array}$ & $\begin{array}{l}4,11(0,46) \\
3,88(0,37)\end{array}$ & $\begin{array}{r}40 \\
162\end{array}$ & $\begin{array}{l}3,92(0,42) \\
3,78(0,35)\end{array}$ \\
\hline $\begin{array}{l}\text { Naturalidade } \\
\text { Distrito Federal }^{\mathrm{e}} \\
\text { Outro local }^{\mathrm{f}}\end{array}$ & $\begin{array}{l}83 \\
13\end{array}$ & $\begin{array}{l}4,12(0,41) \\
3,81(0,32)\end{array}$ & $\begin{array}{l}74 \\
28\end{array}$ & $\begin{array}{l}3,95(0,39) \\
3,88(0,43)\end{array}$ & $\begin{array}{r}119 \\
83\end{array}$ & $\begin{array}{l}3,86(0,37) \\
3,74(0,36)\end{array}$ \\
\hline Total $^{\mathrm{g}}$ & 96 & $4,08(0,41)$ & 102 & $3,93(0,40)$ & 202 & $3,81(0,37)$ \\
\hline
\end{tabular}

* Análise de variância (one-way) por diferentes fatores. Teste robusto de igualdade de médias (Welch, estatística F de distribuição assintótica).

(a) $\mathrm{F}=5,3(2 ; 102,8) \mathrm{P}=0,007 ;$ (b) $\mathrm{F}=11,0(2 ; 95,7) \mathrm{P}<0,001$.

As médias do IRA mostraram diferenças significantes segundo o ano de ingresso, independentemente do efeito da forma de ingresso (MLG, procedimento unianova). Observou-se tendência de elevação do rendimento ao longo dos seis anos (teste de contraste polinomial: termos linear e qua- (c) $\mathrm{F}=2,4(2 ; 48,7) \mathrm{P}=0,104 ;$ (d) $\mathrm{F}=21,0(2 ; 9,7) \mathrm{P}<0,001$.

(e) $\mathrm{F}=10,9(2 ; 162,1) \mathrm{P}<0,001$; (f) $\mathrm{F}=1,3(2 ; 29,9) \mathrm{P}=0,288$.

(g) $\mathrm{F}=15,4(2 ; 199,4) \mathrm{P}<0,001$.

drático significantes). As médias de rendimento dos subgrupos anuais de avaliação seriada foram consistentemente maiores do que as médias de rendimento das outras formas de ingresso, embora as diferenças tenham sido significantes apenas no quarto e no quinto ano de ingresso (Tabela 6).

TABELA 6

Diferenças no Índice de Rendimento Acadêmico (IRA) do primeiro ano entre todos os alunos de Medicina agrupados por ano de ingresso e por forma de ingresso no curso $(\mathrm{N}=400)$

\begin{tabular}{c|c|c|c|c}
\hline Ano de ingresso & $\begin{array}{c}\text { Forma de Ingresso } \\
\text { Avaliação Seriada }\end{array}$ & $\mathbf{1}$ vestibular & 20 vestibular & Total \\
\hline $1(\mathrm{n}=57)$ & $3,97(0,35)$ & $3,63(0,50)$ & $3,73(0,38)$ & $3,75(0,42)$ \\
\hline $2(\mathrm{n}=70)$ & $3,91(0,41)$ & $3,86(0,37)$ & $3,74(0,38)$ & $3,81(0,39)$ \\
\hline $3(\mathrm{n}=71)$ & $4,07(0,57)$ & $3,99(0,32)$ & $3,93(0,39)$ & $3,98(0,32)$ \\
\hline $4(\mathrm{n}=68)$ & $4,23(0,37)$ & $4,15(0,30)$ & $3,80(0,42)$ & $3,96(0,43)$ \\
\hline $5(\mathrm{n}=67)$ & $4,21(0,38)$ & $4,03(0,37)$ & $3,87(0,26)$ & $3,92(0,31)$ \\
\hline $6(\mathrm{n}=67)$ & $4,05(0,28)$ & $3,86(0,41)$ & $3,81(0,37)$ & $3,90(0,40)$ \\
\hline Total $(\mathrm{N}=400)$ & $4,08(0,41)$ & $3,93(0,40)$ & \\
\hline
\end{tabular}

MLG, unianova. Médias do IRA por ano de ingresso: $\mathrm{F}=3,8 \mathrm{df}(5,392) \mathrm{P}=0,002$; teste de contraste polinomial: $\mathrm{P}=0,002$ (termo linear), $\mathrm{P}=0,005$ (termo quadrático). 
A contribuição relativa dos diferentes fatores para a variabilidade do IRA ao término do primeiro ano foi analisada mediante o procedimento unianova do Modelo Linear Geral. A Tabela 7 mostra os efeitos (between-subjects) principais. Valoração do aprendizado (QVC), idade, sexo (feminino vs. masculino), naturalidade (DF vs. outra), ano de ingresso (1999 a 2004) e forma de ingresso (seriado, primeiro vestibular ou segundo vestibular) explicam em conjunto $24 \%$ da variabilidade do IRA, expressa no teste $e t a^{2}$. Interações entre os fatores, motivação para prosseguir os estudos e autoconfiança não contribuíram significantemente para a variabilidade. As parcelas mais importantes na explicação da variabilidade do índice de rendimento no primeiro ano são atribuíveis ao sexo, ao ano de ingresso e à valoração do aprendizado realizado. A contribuição específica da forma de ingresso (independente de todas as outras variáveis) teve pequena magnitude $(2,3 \%)$ na atribuição da variabilidade.

\section{TABELA 7}

Modelo Linear Geral, unianova. Teste de efeitos (between-subjects) de diferentes fatores (sexo, naturalidade, forma de ingresso e ano de ingresso no curso), ajustados para co-variáveis de idade e Valoração do Curso (QVC), no Índice de Rendimento Acadêmico (IRA) de alunos de Medicina ( $=400)$

\begin{tabular}{|c|c|c|c|c|c|c|}
\hline Fonte de Variação & Soma de Quadrados (SQ) & $\mathrm{df}$ & Média (MQ) & $\mathbf{F}$ & Significância & $\mathbf{E t a}^{2}$ \\
\hline Modelo corrigido & 15,75 & 11 & 1,43 & 11,38 & 0,000 & 0,244 \\
\hline Intercepto & 25,90 & 1 & 25,90 & 205,90 & 0,000 & 0,347 \\
\hline Forma de ingresso & 1,12 & 2 & 0,56 & 4,47 & 0,012 & 0,023 \\
\hline Sexo & 3,32 & 1 & 3,32 & 26,40 & 0,000 & 0,064 \\
\hline Naturalidade & 1,21 & 1 & 1,21 & 9,59 & 0,002 & 0,024 \\
\hline Ano de Ingresso & 2,50 & 5 & 0,50 & 3,97 & 0,002 & 0,049 \\
\hline Idade & 1,38 & 1 & 1,38 & 10,98 & 0,001 & 0,028 \\
\hline QVC & 1,51 & 1 & 1,51 & 11,99 & 0,001 & 0,030 \\
\hline Erro & 48,80 & 389 & 0,13 & & & \\
\hline Total & 6164,71 & 400 & & & & \\
\hline Total corrigido & 64,56 & 399 & & & & \\
\hline
\end{tabular}

Variável de desfecho: Índice de Rendimento Acadêmico (IRA), ao término do primeiro ano. Coeficiente de determinação (R²) ajustado $=0,223$.

Dados de 191 alunos (seis turmas), que completaram nove períodos do curso até o segundo semestre de 2005, mostram a evolução do rendimento acadêmico. As médias do IRA foram significantemente diferentes entre os grupos de ingresso em três momentos de medida: ao término do segundo, do

\footnotetext{
* O teste eta-quadrado é uma medida de magnitude do efeito (no caso, efeito dos diferentes
} fatores explicitados na variabilidade do indice de rendimento acadêmico). sexto e do nono períodos do fluxo curricular (Tabela 8). O tamanho do efeito (ES) relativo ao índice de rendimento, na comparação entre avaliação seriada e primeiro vestibular, elevou-se discretamente entre a primeira e a terceira medida: ES $=0,35$ (na primeira), $\mathrm{ES}=0,37$ (na segunda) e $\mathrm{ES}=0,43$ (na terceira). Na comparação entre avaliação seriada e segundo vestibular, a elevação foi bem mais expressiva: $\mathrm{ES}=0,57$ (na primeira), $\mathrm{ES}=0,83$ (na segunda) e $\mathrm{ES}=0,91$ (na terceira medida). 
TABELA 8

Evolução do Índice de Rendimento Acadêmico (IRA) cumulativo entre alunos de seis turmas consecutivas, agrupados por forma de ingresso no Curso de Medicina $(\mathrm{N}=191)^{*}$

\begin{tabular}{c|c|c|c}
\hline Forma de ingresso & $\begin{array}{c}\text { IRA } \\
\text { 2 semestres } \\
\text { Média (dp) })^{\mathbf{a}}\end{array}$ & $\begin{array}{c}\text { 6 semestres } \\
\text { Média (dp) }^{\mathbf{b}}\end{array}$ & $\begin{array}{c}\text { 9 semestres } \\
\text { Média (dp) }\end{array}$ \\
\hline Avaliação seriada $(\mathrm{n}=43)$ & $4,01(0,44)$ & $4,08(0,27)$ & $4,10(0,24)$ \\
\hline Primeiro vestibular $(\mathrm{n}=52)$ & $3,88(0,37)$ & $3,96(0,27)$ & $4,00(0,23)$ \\
\hline Segundo vestibular $(\mathrm{n}=96)$ & $3,80(0,37)$ & $3,82(0,29)$ & $3,87(0,26)$ \\
\hline
\end{tabular}

Sete casos excluídos no seguimento (dois por créditos incompletos, cinco por evasão).

* Análise de variância (one-way) por forma de ingresso, teste F.

(a) IRA de dois semestres: $\mathrm{F}(2,149)=6,3 \mathrm{P}=0,020$.

(b) IRA de seis semestres: $\mathrm{F}(2,149)=12,1 \mathrm{P}<0,001$.

(c) IRA de nove semestres: $\mathrm{F}(2,149)=15,3 \mathrm{P}<0,001$.

\section{DISCUSSÃO}

No cotejo com o exame vestibular, o processo de avaliação seriada selecionou proporções maiores de indivíduos de faixa etária mais jovem e de naturalidade do próprio Distrito Federal, sem efeito expressivo na distribuição sexual, no acumulado do tempo de estudo. Comparações de composição do alunado da época do estudo com alunados de épocas consecutivas anteriores sugerem que o processo de avaliação seriada provavelmente acelerou a tendência de seleção de indivíduos provenientes do Distrito Federal, mas atenuou a tendência anterior de seleção da faixa etária acima dos 18 anos. A tendência referente à faixa etária deve ser situada na perspectiva do estoque crescente de candidatos ao curso, inferido pelo aumento da demanda (número de candidatos por número de vagas).* O efeito eventual da demanda reprimida (não compensada pelo aumento de vagas) seria a elevação da média de idade dos candidatos ao vestibular, mas não dos candidatos à avaliação seriada.

As diferenças observadas entre as formas de ingresso quanto ao rendimento acadêmico eram previsíveis, tendo em conta que o processo de admissão na época do estudo constituiu uma seqüência temporal de filtros seletivos, em que parcelas crescentes de candidatos participaram da etapa anterior sem obter classificação. De qualquer forma, o efeito diferencial do processo seletivo no rendimento acadêmico, embora de pequena magnitude, se mostrou duradouro ao longo de nove

No último qüinqüênio, houve elevação da demanda por ingresso via vestibular, enquanto a demanda por ingresso via avaliação seriada permaneceu estável. semestres de acompanhamento. Essa evidência sugere que tal efeito esteja associado a uma característica estável do aprendiz (e.g., capacidade mental geral), além do conhecimento acumulado.

Entretanto, a predição de sucesso acadêmico no Curso de Medicina abrange múltiplos fatores, tais quais fatores cognitivos (conhecimento, capacidade mental geral), fatores nãocognitivos (incluindo personalidade, estilo de aprendizagem) e fatores demográficos (particularmente sexo) ${ }^{11,12}$. Boa parte da variação observada no rendimento acadêmico no primeiro ano pode ser atribuída às diferenças de sexo, idade, naturalidade, ano de ingresso e valoração do aprendizado realizado. Tais efeitos poderiam estar expressando diferenças psicológicas e educacionais entre os distintos subgrupos. As alunas, por exemplo, teriam mais flexibilidade de adaptação aos desafios do primeiro ano, enquanto indivíduos mais jovens podem ser mais competitivos (dedicar mais esforço) na busca de realização pessoal. A valoração do aprendizado realizado implica motivação intrínseca pelos estudos e sintonia com o ambiente educacional, enquanto a relação do rendimento com o ano de ingresso poderia derivar do próprio incentivo gerado pelo PAS.

O efeito da naturalidade na relação entre forma de ingresso e índice de rendimento é instigante. No contexto do estudo, o atributo naturalidade serve como representação da facilidade de acesso e relevância da preparação para admissão na universidade. Alunos escolarizados no Distrito Federal, mormente em escolas mais qualificadas, estariam em vantagem relativa em termos de acesso ao programa de avaliação seriada e de prontidão educacional ou valoração do estudo. A 
evidência de diferenças associadas à naturalidade no rendimento obtido após o ingresso no curso sugere que as características pessoais implicadas nesse atributo demográfico teriam efeito duradouro no progresso acadêmico dos estudantes.

$\mathrm{Na}$ análise das condições para ingresso na escola médica, McGaghie identificou três metas de curto prazo para a admissão de candidatos ao curso: (a) seleção de candidatos com alta probabilidade de graduação; (b) avaliação da adequação psicológica e estabilidade emocional dos candidatos; e (c) seleção de candidatos que se adaptem aos propósitos da instituição e que agreguem valor ao ambiente educacional ${ }^{1}$.

Alguns achados são sugestivos no exame dos resultados do estudo sob essa tríplice perspectiva. Tanto a adesão ao curso quanto o rendimento acadêmico foram consistentemente mais elevados no grupo selecionado por avaliação seriada. Ambos os efeitos realçam o mérito acadêmico entre os participantes deste grupo, embora o primeiro efeito seja trivial em face do baixo índice de evasão no curso.

A agregação de valor é um efeito potencialmente importante do processo de seleção, mas os dados a respeito não foram explorados neste trabalho. Por outro lado, ainda que adequação psicológica não faça parte do processo de seleção, nem tenha sido avaliada no estudo, chama a atenção a inexistência de diferenças significativas entre os grupos de ingresso nas atitudes dos estudantes em face do aprendizado no curso. Alunos selecionados por avaliação seriada mostraram índices de adesão, graus de motivação para prosseguir nos estudos e níveis de autoconfiança equivalentes aos dos colegas selecionados nos exames vestibulares. Em particular, a adaptação inicial ao curso - revelada na valoração do aprendizado (QVC), que reflete as percepções sobre o valor e o significado do aprendizado no primeiro ano - não diferiu substancialmente entre os integrantes dos grupos de ingresso. Em conjunto, esses achados não conferem uma distinção de perfil psicológico entre os grupos de ingresso no tocante à orientação de aprendizagem.

Os resultados são compatíveis, ao menos em parte, com os objetivos do PAS, na medida em que os achados do estudo sugerem maior influência das diretrizes do programa no sistema educacional do Distrito Federal. Esse influxo é revelado pela ampliação da proporção de oriundos deste município no alunado e, particularmente, pelo incremento do rendimento acadêmico no curso após a introdução do programa, especialmente entre os naturais do Distrito Federal (dados não publicados).

O estudo tem várias limitações associadas ao próprio tipo de delineamento. Dentre diversos tipos de ameaças à validade interna, o principal diz respeito a atributos dos alunos potencialmente associados ao rendimento acadêmico, a exemplo de sexo, motivação, auto-eficácia acadêmica e auto-regulação na aprendizagem ${ }^{13}$. As duas últimas características, que podem diferir entre os grupos de ingresso, não foram aferidas.

Em aditamento, ainda que a generalização dos seus resultados para outras instituições seja problemática, o estudo descortina a utilidade da auditagem de experiências de mudanças no processo de admissão, pouco divulgadas na literatura6,14. Os autores acreditam, também, que o estudo reaviva a questão da seleção específica de candidatos ao Curso de Medicina, tendo em conta a enorme demanda de candidatos, os desideratos emergentes na formação médica, os efeitos de ações afirmativas, a pressão por controle social e outros fatores de influência.

Em suma, uma premissa da instituição do PAS na Universidade de Brasília foi a busca da avaliação da aprendizagem significativa, a qual poderia resultar na seleção de estudantes com perfil diferenciado em termos de motivação e orientação de aprendizagem, além do mérito acadêmico. Os achados do estudo sugerem que o processo de avaliação seriada tem privilegiado discretamente a aptidão acadêmica, em termos de rendimento cognitivo, no âmbito do Curso de Medicina. Em acréscimo, estima-se que essa forma de seleção tenha modificado tendências históricas observadas na composição demográfica do alunado do Curso de Medicina a partir da implantação do novo currículo em 1988, acarretando efeitos no próprio rendimento acadêmico.

\section{REFERÊNCIAS}

1. McGaghie WC. Student selection. In: Norman GR, Van der Vleuten C O M, Newble DI, eds. International Handbook of Research in Medical Education. Dordrecht: Kluwer; 2002 p. 303-335.

2. Brasil. Conselho Nacional de Educação - Conselho Pleno. Parecer n. CP 98/99, aprovado em 06 de julho de 1999. Regulamentação de Processo Seletivo para acesso a Cursos de graduação de Universidades, Centros Universitários e Instituições Isoladas de Ensino Superior. Brasília: Conselho Nacional de Educação;1999.

3. Schlichting AM, Soares DHP, Bianchetti L. Vestibular seriado - análise de uma experiência em Santa Catarina. Biologia \& Sociedade 2004; 16(2):114-26.

4. Brasil. Conselho Nacional de Educação. Constituição 1988. Texto Constitucional de 05 de outubro de 1988 com as alterações adotadas pelas Emendas Constitucionais no 1 / 1992 a 30/2000 e Emendas Constitucionais de Revisão nº 1 a 6/1994. Brasília: Ed. Atual; 2000. 
5. Universidade de Brasília - CESPE. Programa de Avaliação Seriada (PAS). Disponível em: www.cespe.unb.br/pas/oquepas/ principios/principiospas.htm [Acesso em: 24/05/2005].

6. Binia A, Ciccarelli, AS, Ortiz AM. Experience with a selection method for admittance to the Universidad Nacional de Cuyo Medical Sciences School. In: Scherpbier AJJA, Van der Vleuten CPM, Rethans JJ, Van der Steeg AFW, eds. Advances in Medical Education. Dordrecht: Kluwer;1995. p. 636-637.

7. Nehari M, Bender H. Meaningfulness of a course experience: a measure for educational outcomes in higher education. Higher Education 1978; 7:1-11.

8. Sobral DT. Estilos de aprendizagem dos estudantes de medicina e suas implicações. Rev Bras Educ Méd 2005; 29: 5-12.

9. Fraenkel JR, Wallen NE. How to Design and Evaluate Research in Education. New York: McGraw-Hill; 2003.

10. Howell DC. Statistical Methods for Psychology 5. ed. Pacific Grove (CA): Duxbury: 2002.

11. Ferguson E, James D, Madeley L. Factors associated with success in medical school: systematic review of the literature. Brit Med J 2002; 324: 952-956.
12. Ferguson E, James D, O'Hehir F, Sanders A. Pilot study of the roles of personality, references, and personal statements in relation to performance over five years of a medical degree. Brit Med J 2003; 326: 429-34.

13. Pajares F. Self-efficacy beliefs in academic contexts: an outline. Emory University; 2002. Disponível em: www.emory.edu/education/mfp/efftalk.html [Acesso em: 17/10/2005].

14. Collins JP, White GR, Kennedy JA. Entry to medical school: an audit of traditional selection requirements. Med Educ 1995; 29: 22-28.

\section{Conflito de Interesse}

Declarou não haver.

\section{Endereço para correspondência}

D.T. Sobral

Universidade de Brasília

Faculdade de Medicina - CP 04569

70919-970 - Brasília - DF

e-mail: dtsobral@unb.br, pgo@terra.com.br 